\title{
Failure Analysis and Safety Protection of a Certain Type of Wire Ropes under High-Speed Impact Loads
}

\author{
Zhiqian Ren ${ }^{1}{ }^{*}$, Zhiqiang $\mathrm{Lu}^{2}$, Qiong $\mathrm{Yu}^{2}$ and Yun $\mathrm{Jiang}^{2}$ \\ ${ }^{1}$ Science and Technology on Integrated Logistics Support Laboratory, National University of Defense \\ Technology, Changsha, China \\ ${ }^{2}$ Hunan Sushiguangbo Testing Technology Co.,Ltd, Changsha, China
}

\begin{abstract}
As the key bearing components in engineering projects, wire ropes often suffer from high-speed impact loads in service. In the majority of applications, wire ropes are subjected to the loads with the following characteristics: alternating impact loads, short-time overload, and small movements among adjacent spiral strands. Typical failure modes of wire ropes include fracture, wear, corrosion, geometric failure and thermal failure. In this work, the corresponding failure mechanisms were analyzed. For a certain type of wire ropes, the fracture morphology, surface quality, microstructure and internal defects of steel wires were analyzed, and failure causes were discussed. Accordingly, the precautions in relation to the safe use of wire ropes were proposed.
\end{abstract}

\section{Introduction}

Wire ropes are widely used in many industrial fields because of their large bearing capacity, good flexibility and smooth motions without noise. However, they tend to degrade due to a range of factors including environmental corrosion, uncertain alternating loads, mechanical impacts, wear and high-speed impact stress, resulting in wear, corrosion and wire fracture, hence decreased strength. With the reduction of strength, wire ropes may suddenly break, inducing security incidents such as production pauses and even casualties. Therefore, conducting the failure analysis of wire rope is of vital significance on both safety and economy in production.

\section{Characteristics of wire rope loads}

\subsection{Alternating impacts}

Each working cycle of wire ropes includes linear lift, movement through the pulley, coiling on the winding drum and unload drop. Therefore, the force on each steel wire is essentially

\footnotetext{
${ }^{*}$ Corresponding author: rzq_rtl@nudt.edu.cn
} 
alternating stress, and the wire ropes are subjected to fatigue loads and alternating highspeed impact loads [1].

\subsection{Short-time overload}

Wire ropes elongate slightly with the increase of loading, and it may fracture when the load exceeds the elastic limit. Static loads of wire ropes are generally controlled within around $10 \% \sim 20 \%$ amount of the minimum breaking strength, referred to as safe working loads. However, wire ropes are in a state of motions during use. In addition to the cargo weight, rope self-weight and other static loads, wire ropes also bear the dynamic loads caused by impacts and the acceleration of movements, the additional load caused by bending, and the resistance load caused by friction, etc. The actual safety factor would decrease when other loads apart from static loads increase, and wire ropes may be subjected to short-time overload in consequence [2].

\subsection{Small movements among adjacent spiral strands}

The structure of a typical wire rope is shown in Fig. 1, where the spiral strands are mutually twisted. The steel wires in the same spiral strand deform synchronously, but the deformations of adjacent spiral strands are slightly different, inducing relative sliding among strands and wires, which means that the steel wires are under fretting wear environment in actual service [3].

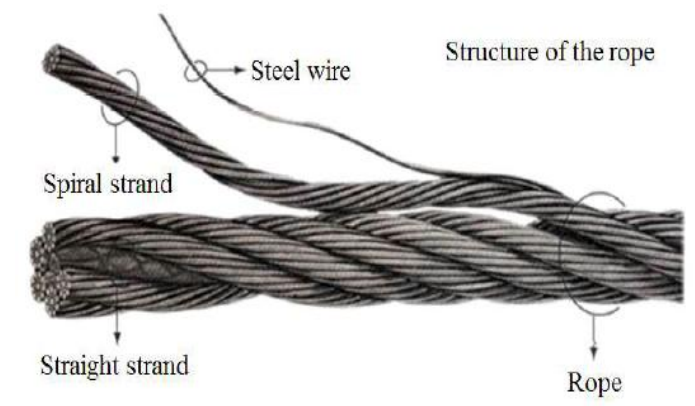

Fig. 1. Schematic diagram of the structure of a typical wire rope.

\section{Typical failure modes and failure mechanisms of wire ropes}

Some scrapped wire ropes were detached and analyzed, and the failure modes of wire rope were classified into three categories according to the external form, i.e., fracture, excessive deformation and surface damage. These failure modes and the corresponding failure mechanisms are summarized and discussed as follows.

\subsection{Fracture}

Fracture is the most common failure mode of wire ropes. Fracture failure can be divided into three kinds according to the special structure of wire ropes, i.e. fracture of steel wires, fracture of spiral strands and fracture of wire ropes. Fracture of wire ropes and spiral strands result from steel wire fracture, and therefore analyzing the failure mechanisms of steel wires fracture is the key to reveal the fracture of wire ropes.

Failure modes of steel wire fracture were widely studied in previous studies, and were classified into three kinds [4], i.e., (1) fracture from a single applied load, which is induced 
when a single applied load exceeds the bearing capacity of steel wires; (2) fatigue fracture resulting from alternating stress; (3) fracture from environmental mediums, which is low stress brittle fracture caused by environmental medium and applied stress.

Typical fracture morphologies are summarized from a great deal of fractured steel wires as shown in Fig. 2. Fracture from a single applied load shows tensile or shear breakage characteristics. A steel wire would fracture when the axial load exceeds its breaking strength, and the wire near the fractured region would elongate with the reduction of its diameter, resulting in the typical cup-cone fracture morphology, as shown in Fig. 2(a). Shear fracture is induced by the load perpendicular to the axial direction, and the angle between the fracture surface and the axis is $45^{\circ}$, as shown in Fig. 2(b). Fatigue is also commonly found to be the main reason for fractured steel wires. In traction and hoisting wire ropes, the fatigue fracture of steel wires is mainly caused by alternating bending under stress through the pulley and winding drum in consequence of the fatigue of metals. The fatigue fracture usually occurs among the outer steel wires in the worst bending side, and the fracture morphology is relatively flush, as shown in Fig. 2(c).

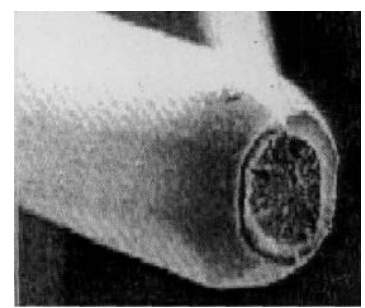

(a) Cup-cone fracture

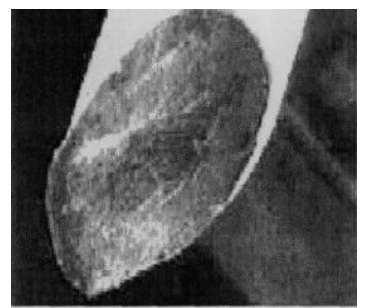

(b) Shear fracture

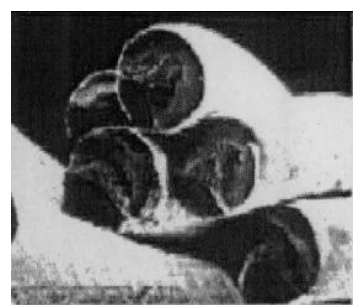

(c) Fatigue fracture

Fig. 2. Typical fracture morphologies of fractured steel wires.

\subsection{Wear}

There are micro relative movements on the contact surface between the surface of a steel wire and pulley, winding drum or the adjacent steel wires, inducing the separation of surface material particles from the substrate due to the integrative effect of mechanical, physical and chemical processes. This would further lead to the change of shapes, dimensions and weight of wire ropes, i.e. wear of wire ropes. The phenomena of dimension reduction, surface state changes and even loss of functions are all classified as wear failure. Wear failure is a gradual process, which is not as sudden as fracture failure accidents. However, wear usually results in the decrease of bearing areas and the increase of fatigue sensitivity, which may further cause fracture failure, especially in corrosive mediums, as wear can accelerate corrosion. The wear failure can be classified into two kinds [5], i.e., mechanical wear and plastic wear. The main difference between the two forms is that the latter shows the material deformation or displacement without material loss phenomenon.

\subsubsection{Mechanical wear}

The reason for the mechanical wear of external steel wires lies in the fact that the steel wire constantly contacts with rope race, lifting hook, ground, etc. The mechanical wear is especially obvious on the contact areas between the wire ropes and pulley under acceleration and deceleration operations, leading to the decrease of wire diameters, loading areas and capacity. In addition, lack of lubrication, existence of sand and gravel grains between spiral strands can also aggravate the mechanical wear.

\subsubsection{Plastic wear}


Surface wear of wire ropes caused by vibration, impact and internal squeezing is called plastic wear, i.e. localized wear. Strikes from other objects on the wire rope coiled on a winding drum and mutual winding of wire ropes would lead to plastic wear. With wire ropes repeatedly curved, the interaction forces and slips between steel wires would occur, and then the contact stress between spiral strands increases, leading to localized indentation and plastic wear of wire ropes [6]. Although the cross-sectional area decreases slightly, surface materials in the localized squeezing place would harden, which tends to induce cracks or even fracture.

\subsection{Corrosion}

As wire ropes mainly serve in relatively severe working environments such as outside and marine environments, corrosion occurs due to the reactions with environmental mediums. Fracture, wear and corrosion are the main damage types for wire ropes. However, unlike fracture, both of wear and corrosion are gradual processes, and they usually interact with each other, inducing early failure of wire ropes. Meanwhile, corrosion can provide conditions for fracture, and accelerates or even directly causes fracture.

Wire rope corrosion can be classified into two categories [7]. The first kind is general corrosion due to contact with air, leading to chemical corrosion and electrochemical corrosion. Chemical corrosion occurs when wire rope is exposed in acidic environment, and electrochemical corrosion occurs in humid air environment. The second kind is localized corrosion such as pitting corrosion, by which holes or cavities are produced in the place where the coating breaks down and comes off. The corrosion pit gradually deepens on the wire rope surface, inducing stress concentration, fatigue crack and decrease of strength and flexibility. The stress concentration will accelerate the extension of cracks, and finally result in mechanical rupture.

\subsection{Other failure modes}

\subsubsection{Geometric failure}

Geometric failure mode occasionally occurs to wire ropes because of their special structures, as shown in Fig. 3.

The phenomena of loose strand and knotting are usually found in hoisting wire ropes, as shown in Fig. 3(a) and Fig. 3(b). Rotation against the twist direction and twist around its axis are the most common reasons for the failure [8].

Steel wire extrusion is also a common geometric failure mode, as shown in Fig. 3(c). It is caused by insufficient interspace between steel wires in a spiral strand. The steel wire cannot move with the adjacent wires when the interspace is insufficient, leading to overload or yielding. The interspace of outside spiral strands will be insufficient if the diameter of the rope core is too small, resulting in the damage of spiral strands [9].

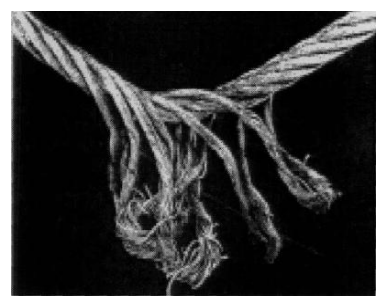

(a) loose strand

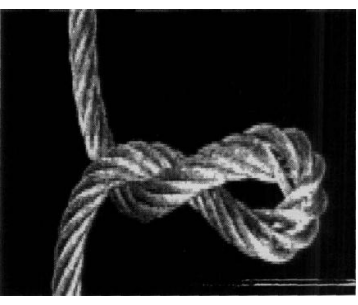

(b) knotting

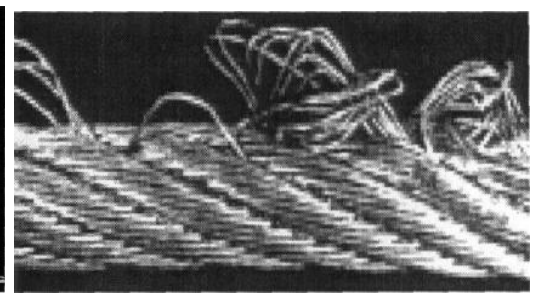

(c) steel wire extrusion

Fig. 3. Typical geometric failure of wire ropes. 


\subsubsection{Thermal failure}

Thermal failure mainly results from the formation of martensite and heat corrosion. Martensite is a brittle metal structure, a very thin layer of which can be usually found on the convex part of the wire. Electric arc caused by electric welding will induce the formation of martensite on the wire rope surface. This martensite layer tends to fracture when the wire rope bends, because it would lead to stress concentration, strength reduction and the formation of fatigue crack [10]. The fatigue crack extends rapidly, inducing abrupt fracture of wire ropes in service.

As it is a good conductor of heat, localized temperature of wire ropes will increase when it works under the environment of high temperatures. Recrystallization would occur to the microstructure of cold drawn wire ropes if the internal temperature exceeds $300^{\circ} \mathrm{C}$, and then the tensile strength of steel wires would drop to two-thirds of the value at room temperature [11]. Wire ropes will be heated up fast if the heat absorption exceeds thermal dissipation, leading to the heat corrosion.

\section{Failure analysis cases of wire ropes}

\subsection{Fracture morphology of steel wires}

Scanning electron microscope (SEM) morphologies of typical fractured steel wires are shown in Fig. 4. The fracture mechanism of the wire rope is determined to be fatigue fracture judging from the morphologies of the fracture surfaces. A small fraction of fractured steel wires in the service of a wire rope would not cause the abandonment of the whole wire rope. If some fractured steel wires that were not cut out in time, and the rupture region would consequently be rubbed to a flat area, as shown in Fig. 4(a). Torn areas can still be clearly observed in some fatigue fracture surfaces, as shown in Fig. 4(b) and Fig. 4(c). Through analyzing the fracture morphologies of the steel wires, it is revealed that most fatigue fracture is caused by high nominal stress, and stress concentration exists in crack source on a small part of the fracture surface, as shown in Fig. 4(c).

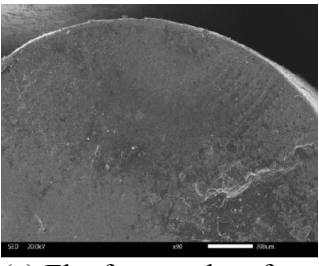

(a) Flat fractured surface

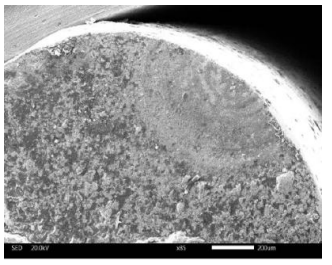

(b) Torn fractured surface

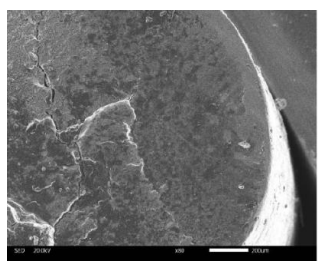

(c) Fractured surface showing stress concentration

Fig. 4. Typical fracture morphologies of fractured steel wires.

\subsection{Surface quality of steel wires}

Typical morphology of steel wire surface is shown in Fig. 5, in which a large number of microcracks exist on the surface of the steel wire. The reason for this phenomenon is that the fatigue cracks mostly originate from the surface or sub-surface of steel wires. Surface quality of steel wires has important effect on its fatigue resistance. The surface cracks would continue to extend inward and finally induce the fracture of the steel wire under applied loading stress. 


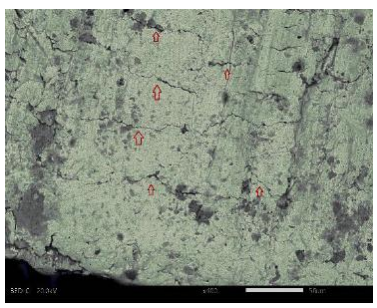

Fig. 5. Typical morphology of steel wire surface.

By observing the longitudinal section of the steel wire shown in Fig. 6, it can be seen that the coating thickness of the new steel wire is larger than that of the old one due to the wear of steel wires in service. In addition, the galvanized layers of both steel wires are uneven, which may affect the surface quality of wire ropes, and even induce localized severe wear and fatigue fracture. Therefore, reasonable measures should be adopted in the production of galvanized layers in order to improve the surface quality of wire ropes.

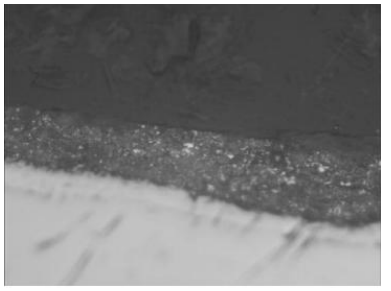

(a) a new wire rope

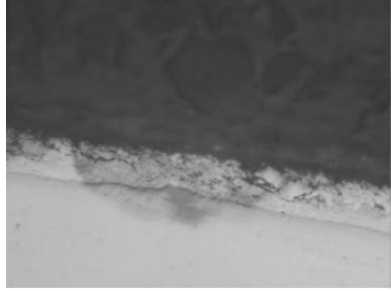

(b) an old wire rope

Fig. 6. Optical microscopy of longitudinal cross-section of steel wires $(\times 500)$.

\subsection{Microstructure of steel wire}

Two different areas on the fatigue fracture surface of a steel wire were chosen to conduct Energy Dispersive Spectrometer (EDS) spot scanning analysis, as shown in Fig. 7. EDS analysis shows that the carbon content of the spot closer to the surface is higher than the other, resulting in higher hardness of the steel wire surface than that of the inner area. When under alternating stress, cracks would firstly originate on the interface close to the steel wire surface between the two steel microstructures, and the fracture of steel wire finally occurs with the crack continuously extending inward.

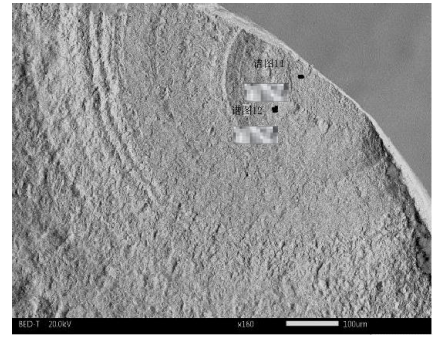

Fig. 7. Locations of EDS spots scanning on the fatigue fracture surface of a steel wire.

Metallographic structures of steel wires after being corroded by $4 \mathrm{wt} . \%$ of nitric acid in alcohol solvent is shown in Fig. 8. It shows that the metallographic structure of the old wire rope is slenderer than that of the new one due to the elongation of the wire rope in actual service. Work hardening phenomenon occurs to the elongated metallographic structure, leading to the increase of brittleness [12]. Scars caused by squeezing between steel wires 
would appear on the surface of steel wires during use. Therefore, the metallographic structure and its orientation near the scar is obviously different from that in other areas, and the fatigue cracks tend to originate in the transition region between the two microstructures. Meanwhile, the regional agglomeration of single black microstructure named cementite and single white microstructure called ferrite exist in both wire ropes, resulting in different performance among different microstructures and initial cracks. The early fatigue fracture of steel wires is strongly related to uneven microstructure [13].

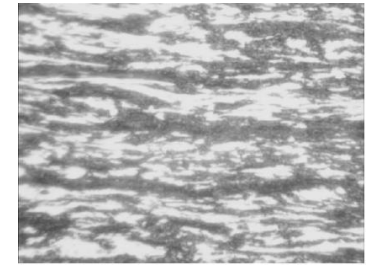

(a) a new wire rope

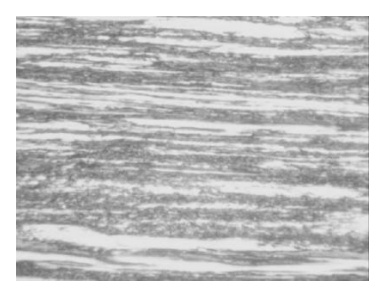

(b) an old wire rope

Fig. 8. Metallographic structures of steel wires $(\times 800)$.

\subsection{Internal defects of steel wires}

Internal defects of steel wires observed by a metallographic microscope are shown in Fig. 9. It can be seen that serious internal discontinuous defects such as slag inclusions or air voids exist in both new and old wire ropes. The defects would become fatigue crack sources, and therefore internal defects of steel wires should be strictly controlled and minimized in the production of wire ropes.

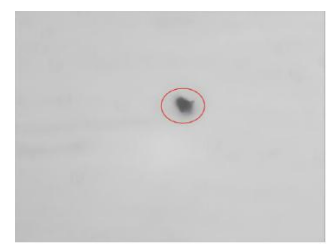

(a) a new wire rope

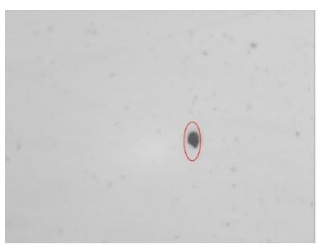

(b) an old wire rope

Fig. 9. Internal defects of steel wires $(\times 500)$.

\section{Precautions for safe use of wire ropes}

Wire ropes often bear high-speed impact loads in actual service. Based on the above analysis of failure modes, failure mechanisms and failure cases, precautions for the safe use of wire ropes in service are summarized as follows:

(1) Frequent sharp change of operating speed will induce high-speed impact loads in actual service. A wire rope may fracture when the high-speed impact loads exceed its allowable working stress. Although high-speed impact loads do not necessarily result in the fracture of wire ropes, it would certainly shorten the service life of wire ropes severely. Therefore, the sharp acceleration, deceleration and emergency braking should be avoided during the use of wire ropes.

(2) Overload operations should be strictly prohibited for wire ropes. Overload operations will obviously increase the wear degree of steel wires, and may cause the fracture of steel wires, which would greatly affect the operation security of wire ropes.

(3) Friction between wire ropes and other objects except pulley under high speed operation should be strictly prohibited, because the friction under high-speed operations 
will generate instantaneous frictional heat, which may lead to the formation of martensite structure on the surface of steel wires and early cracks of steel wires.

(4) Sliding out from the corresponding pulley groove should be strictly prohibited for wire ropes, because it would bring about squeezing deformation, knotting, steel wire fracture and spiral strands fracture, which will shorten the service life of wire ropes or even cause catastrophic consequences.

(5) Disheveled twist should be strictly prohibited for wire ropes. The wire rope on the drum winding should be as orderly as possible; otherwise it will induce mutual squeezing, structural damage and early fracture of steel wires, directly affecting the service life of wire ropes.

(6) Wire ropes should be avoided from water and wet sand in actual service, and they should be used in the environment as dry as possible. Galvanized wire ropes or other anticorrosion wire ropes are recommended to serve in severe service environment. Lubrication is also recommended during use for common wire ropes.

\section{Conclusions}

Wire ropes are often subjected to high-speed impact loads in a range of industrial applications. The loads applied on the wire rope have the following characteristics: alternating impacts, short-time overload, and small movements among adjacent spiral strands. We analyze the typical failure modes of wire ropes in this paper. Based on the analysis of failure modes, failure mechanisms and failure cases, the precautions for the safe use of wire ropes have been summarized. The primary precaution is that the sharp acceleration, deceleration and emergency braking should be avoided during the use of wire ropes.

\section{References}

1. J. Ma, S.R. Ge, D.K. Zhang, J. China. Univ. Min. Techno. 18, 475 (2008).

2. O.A. Goroshko, Int. Appl. Mech. 43, 64 (2007).

3. D.G. Wang, D.K. Zhang, S.R. Ge, Ind. Lubr. Tribol. 65, 436 (2013).

4. D. Elata, R. Eshkenazy, M.P. Weiss, Int. J. Solids Struct. 41,1157 (2004).

5. D.K. Zhang, S.R. Ge, D.S. Xiong, Tribol. 21, 362 (2001).

6. Y. Shen, D.K. Zhang, D.G. Wang, Tribol. 30, 404 (2010).

7. D.G. Wang, D.K. Zhang, W.J. Zhao, et al., Mater. Sci. Eng. A 596, 80 (2014).

8. K. Kumar, J. Botsis, J. Appl. Mech. 68, 432 (2001).

9. X.H. Lu, Z.P. Chen, S.G. Wang, Met. Prod. 39, 1 (2013).

10. J.M. Han, L.Y. Wang, W.Y. Chen, J. Taiyuan Uni. Techno. 31, 218 (2000).

11. F.G. Liu, Met. Heat Treat. 9, 42 (1999).

12. M. Giglio, A. Manes, Eng. Fail. Anal. 12, 549 (2005).

13. K. Kumar, J.E. Cochran. J. Appl. Mech. 54, 898(1987). 